\title{
Oculopharyngodistal myopathy with coexisting histology of systemic neuronal intranuclear inclusion disease: Clinicopathologic features of an autopsied patient harboring CGG repeat expansions in $L R P 12$
}

\author{
Rie Saito ${ }^{1}$, Hiroshi Shimizu ${ }^{1 *}$, Takeshi Miura ${ }^{2,3}$, Norikazu Hara ${ }^{4}$, Naomi Mezaki ${ }^{2,3}$, Yo Higuchi ${ }^{3,4}$, Akinori Miyashita ${ }^{4}$, \\ Izumi Kawachi ${ }^{3,5}$, Kazuhiro Sanpei ${ }^{2}$, Yoshiaki Honma ${ }^{2}$, Osamu Onodera ${ }^{3}$, Takeshi Ikeuchi ${ }^{4}$ and Akiyoshi Kakita ${ }^{1}$
}

Keywords: Oculopharyngodistal myopathy, Neuronal intranuclear inclusion disease, Oculopharyngeal myopathy with leukoencephalopathy, Noncoding CGG expansions, Neuropathology

Unstable tandem repeat expansions are important genetic motifs that underlie various neurological disorders. Two interesting aspects of noncoding CGG expansions have recently attracted attention: pleiotropy in which expanded CGG repeats in NBPF19 cause two disorders that were formerly unlinked, namely neuronal intranuclear inclusion disease (NIID) [2, 4] and essential tremor [6], and the clinical spectrum resulting from similar CGG repeat expansion motifs in different genes, namely NBPF19, LRP12, and LOC642361, which cause NIID, oculopharyngodistal myopathy (OPDM), and a new overlapping disease oculopharyngeal myopathy with leukoencephalopathy (OPML), each designated as NIID1, OPDM1, and OPML1, respectively [2]. Despite such improved understanding of their clinical/genetic aspects, the pathological features of these disorders remain elusive.

Recently, we have experienced a two-generation pedigree of clinically typical OPDM [1] that affected the

\footnotetext{
* Correspondence: hshimizu@bri.niigata-u.ac.jp

'Department of Pathology, Brain Research Institute, Niigata University, 1-757 Asahimachi, Chuo-ku, Niigata 951-8585, Japan

Full list of author information is available at the end of the article
}

father and one of his two children (Fig. 1a); the latter (the proband) was autopsied and shown to harbor CGG repeat expansions in LRP12 (Fig. 1b), establishing the diagnosis of OPDM1. Both the proband and his father developed adult-onset ptosis, distal-dominant slowly progressive myopathy with rimmed vacuoles (RVs) (Fig. 2a-d) [3], dysphagia, and external ophthalmoplegia (Additional file 1). Ultrastructural analysis of the necropsied scalenus muscle revealed tubulofilamentous inclusions in the muscle fiber nuclei [3]; occasionally, two inclusions were observed in one nucleus (Fig. 2e, f). Importantly, these inclusions were undetectable by routine histology or ubiquitin immunohistochemistry. In addition to the muscle pathology, a general autopsy of the proband unexpectedly revealed coexisting NIID histopathology. Almost all organs including the central and peripheral nervous systems, except for the skeletal muscles, showed abundant round, eosinophilic intranuclear inclusions (Fig. 3a) that were positive for ubiquitin (Fig. 3b) and p62 and most frequently detectable in the sympathetic (Fig. 3c) and dorsal root ganglia. In these two regions, 54 and $41 \%$ of neurons possessed p62- 

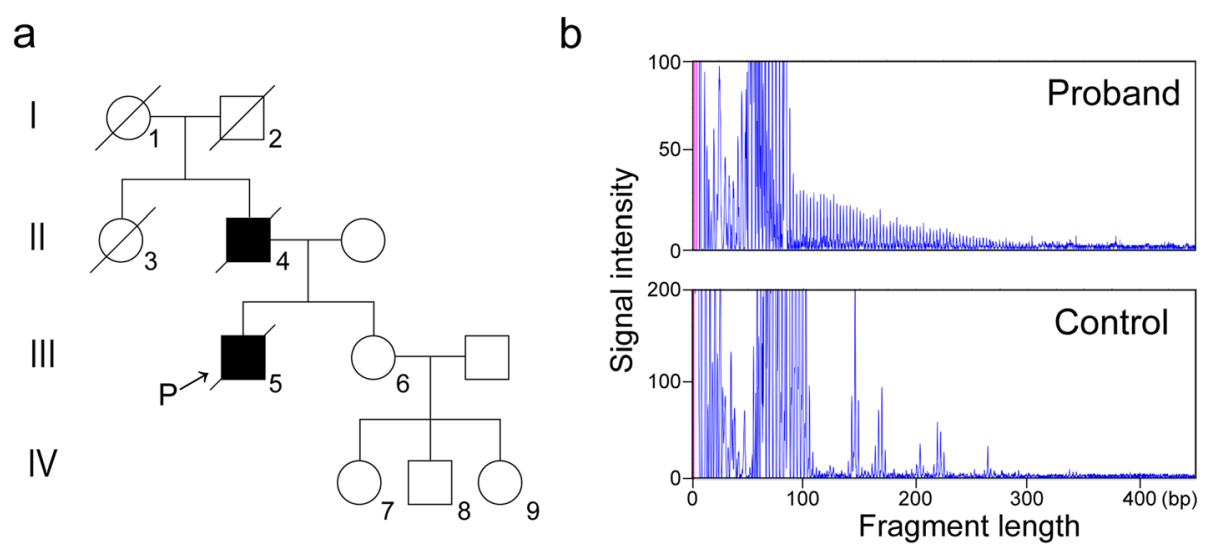

Fig. 1 Detection of CGG repeat expansions in LRP12. a Pedigree of the family. The individuals suffering from OPDM are indicated by solid symbols. The family shows autosomal dominant inheritance. $\mathbf{b}$ Repeat-primed PCR analysis using frozen frontal cortex obtained from the proband (a, III-5) demonstrates CGG repeat expansions in LRP12 (upper panel), whereas no such expansions are evident in the control (lower panel). In the proband, there were no pathological tandem repeat expansions in FMR1, NBPF19, LOC642361/NUTM2B-AS1, or PABPN1, genetically excluding the possibility of fragile $X$ tremor/ataxia syndrome (FXTAS), neuronal intranuclear inclusion disease 1 (NIID1), oculopharyngeal myopathy with leukoencephalopathy 1 (OPML1), or oculopharyngeal muscular dystrophy (OPMD). P, proband

positive nuclear inclusions, and 3 and $5 \%$ possessed two inclusions, respectively. In the brain, these inclusions were observed in neurons (Fig. 3d), astrocytes (Fig. 3e), and Schwann cells. The distribution (Table 1) and ultrastructural features (Fig. 3f, g) of the inclusions were indistinguishable from those in NIID1 $[4,5]$.

These findings suggest that the disease process of OPDM1 is not limited to the oculopharyngeal muscles and can affect various extra-skeletal muscle organs including the central and peripheral nervous systems. Furthermore, the observed ubiquitinated inclusions were indistinguishable from those in NIID1, strongly supporting the hypothesis that such transcribed expanded CGG repeats are commonly involved in the development of OPDM-NIID spectrum disorders, irrespective of the genes where the repeats are located [2].

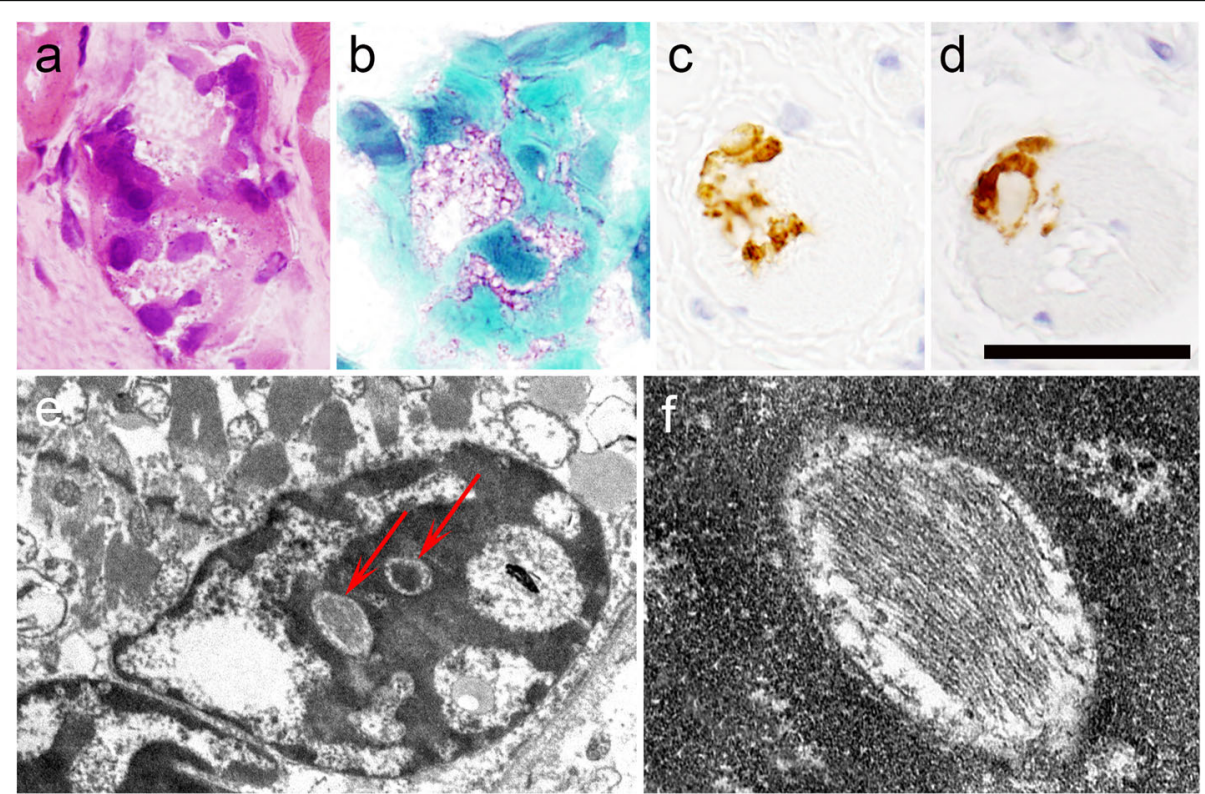

Fig. 2 Pathology of the skeletal muscles. a-d Rimmed vacuoles (RVs). Skeletal muscles show typical RVs on staining with HE (a) and modified Gomori trichrome (b). RVs are positive for p62 (c) and phosphorylated TDP-43 (d). e, $\mathbf{f}$ Intranuclear inclusions of the skeletal muscles. These inclusions were detectable only by electron microscopy (e). They had no obvious limiting membranes and were composed of straight filaments about 13-18 nm in diameter (f). a, b anterior tibial muscle, biopsy; $\mathbf{c}, \mathbf{d}$ striated muscle of the esophagus, necropsy; e, $\mathbf{f}$ scalenus muscle, necropsy. Scale bar: $\mathbf{a}-\mathbf{d}=40 \mu \mathrm{m}, \mathbf{e}=2 \mu \mathrm{m}, \mathbf{f}=500 \mathrm{~nm}$. 


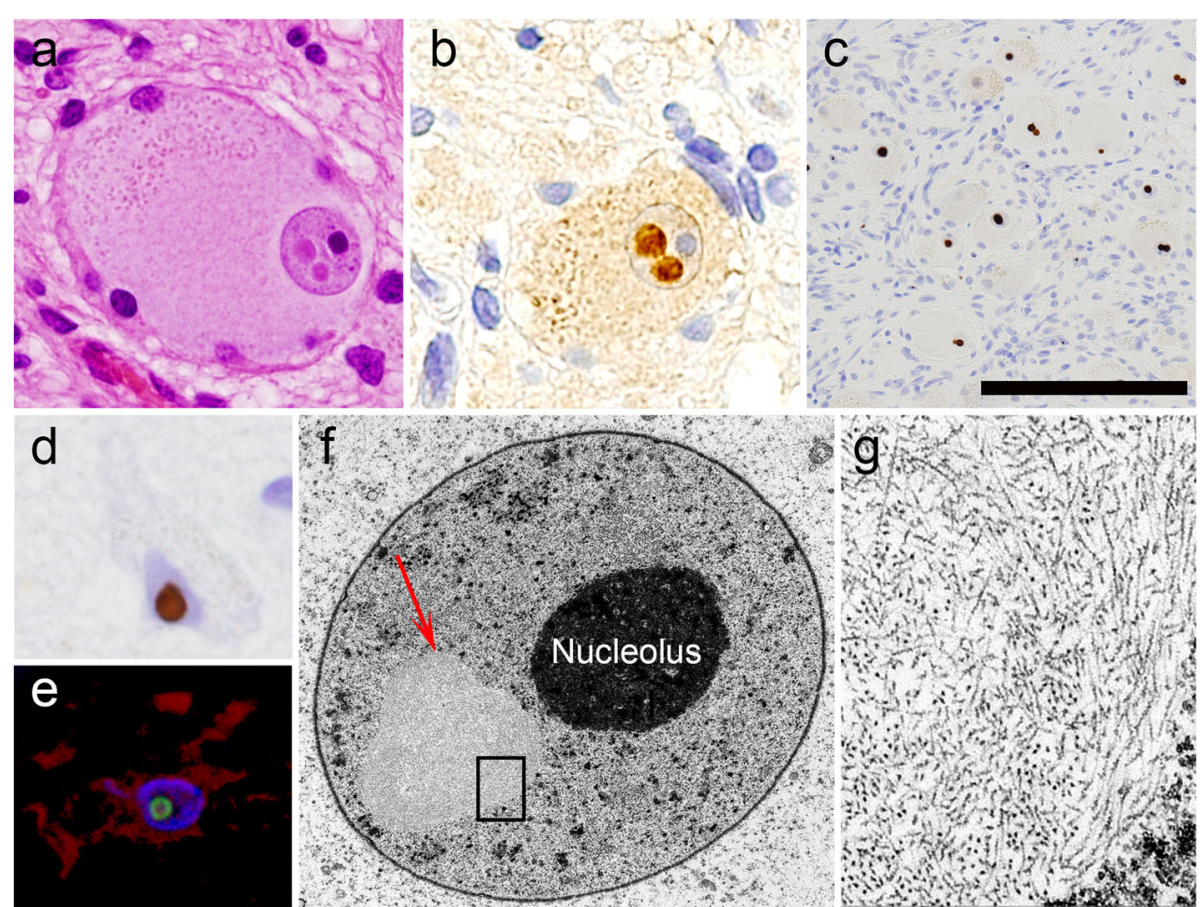

Fig. 3 Intranuclear inclusions in extra-skeletal muscle organs. a-c Sympathetic ganglia. Two eosinophilic neuronal intranuclear inclusions with surrounding halos are evident on HE staining (a). The inclusions are positive for ubiquitin (b). Numerous inclusions are revealed by p62immunohistochemistry (c). d A p62-positive neuronal intranuclear inclusion in the transentorhinal cortex. e A p62-positive intranuclear inclusion (green) in a GFAP-positive astrocyte (red) in the temporal cortex. Double-labeling immunofluorescence. $\mathbf{f}, \mathbf{g}$ Ultrastructure of a sympathetic ganglion neuron containing an intranuclear inclusion (f, arrow). The inclusion is composed of fine filamentous structures without limiting membranes. Scale bar: $\mathbf{a}, \mathbf{b}=40 \mu \mathrm{m} ; \mathbf{c}=150 \mu \mathrm{m} ; \mathbf{d}, \mathbf{e}=25 \mu \mathrm{m} ; \mathbf{f}=5 \mu \mathrm{m} ; \mathbf{g}=750 \mathrm{~nm}$

A major question that naturally arises is whether or not the NIID1-like lesions with intranuclear inclusions described here caused symptoms. The major clinical features of NIID such as dementia, parkinsonism, or neurogenic muscular weakness were not observed in the present pedigree, implying that in OPDM-NIID spectrum disorders, development of clinical symptoms is not merely dependent on the transcribed CGG repeats, but also associated with dysfunction of the mutated genes. Unfortunately, due to agonal brain edema and absence of brain MRI data, we could not determine the presence or absence of cerebral white matter degeneration. In OPDM1 and OPML1, however, lesions in the autonomic nervous system may commonly develop. Three out of six patients of the OPML1 pedigree had gastrointestinal symptoms [2], while the present OPDM1 patient showed the highest density of intranuclear inclusions in autonomic ganglia neurons, although the symptoms were unremarkable. Currently, it remains unclear whether the hypertrophic cardiomyopathy observed in the present OPDM1 patient (Additional file 1), or the rare occurrence of dilated cardiomyopathy in patients with OPDM [7] and OPML1 [2] is also associated with CGG repeat expansions. Further studies are necessary to clarify any extra-muscular organ involvement and associated symptoms that might have been masked by more obvious features relevant to muscle pathology.

Noncoding repeat expansions cause diseases through several mechanisms such as loss of expression of the repeat-containing genes and RNA gain-of-function, the latter including 1) repeat associated non-AUG (RAN) translation that generates toxic, often ubiquitinated aggregation-prone proteins and 2) formation of RNA foci that sequester RNA-binding proteins. In the present autopsied patient with OPDM1, the inclusions in the skeletal muscles were not ubiquitinated and visible only by electron microscopy, whereas those in extra-skeletal muscle organs were ubiquitinated and detectable by routine histology. The mutually exclusive distribution and different natures of these two types of inclusion may reflect a difference in the pathomechanisms whereby they are recruited to skeletal muscles or extra-skeletal muscle organs. Interestingly, like OPDM1, no eosinophilic, ubiquitinated inclusions have been reported in skeletal muscles in OPML1 or NIID1 (Table 2). It would be of great interest if extensive electron microscopy examination revealed that inclusions in skeletal muscles morphologically resembled those observed in OPDM1.

In conclusion, we have presented an OPDM1 pedigree associated with LRP12 including the first reported 
Table 1 Distribution and density of p62-positive intranuclear inclusions in the proband

\begin{tabular}{|c|c|c|c|c|c|c|}
\hline CNS & Neuron & Astrocyte & PNS & Neuron & Schwann cell & \\
\hline Cerebrum & & & Sympathetic ggl & 3 & 2 & \\
\hline Frontal $^{a}$ & 1/n.a. & $1 / 1$ & Dorsal root ggl & 3 & 2 & \\
\hline Motor $^{a}$ & $2 /$ n.a. & $1 / 1$ & Myenteric ggl & 2 & 2 & \\
\hline Temporal $^{a}$ & 2/n.a. & $2 / 1$ & & & & \\
\hline Parietal $^{\mathrm{a}}$ & 1/n.a. & $1 / 1$ & Visceral organs & & & \\
\hline Occipital $^{a}$ & 2/n.a. & $1 / 1$ & Kidney & Renal tubule & Glomerulus & \\
\hline Hippocampus ${ }^{b}$ & $2 / 3 / 1$ & $1 / 1 / 1$ & & 2 & 2 & \\
\hline Amygdala & 2 & 1 & Liver & Hepatocyte & & \\
\hline NBM & 1 & 1 & & 0 & & \\
\hline Caudate/putamen & $1 / 1$ & $1 / 1$ & Pancreas & Acinar cell & Ductal cell & \\
\hline Globus pallidus i/e & $1 / 1$ & $1 / 1$ & & 1 & 1 & \\
\hline Thalamus & 2 & 1 & Adrenal & Cortex & Medulla & \\
\hline Brainstem & & & & 1 & 1 & \\
\hline Midbrain tectum & 2 & 1 & Heart & Muscle cell & & \\
\hline Oculomotor nuc & 2 & 2 & & 1 & & \\
\hline Red nuc & 2 & 2 & Skeletal muscle & Muscle cell & & \\
\hline Substantia nigra & 3 & 2 & & 0 & & \\
\hline Locus ceruleus & 2 & 1 & & & & \\
\hline Facial nuc & 2 & 1 & Somatic cells & Adipocyte & EC & $S M C$ \\
\hline Pontine nuc & 1 & 1 & & 1 & 1 & 2 \\
\hline Hypoglossal nuc & 2 & 0 & & & & \\
\hline 10 & 2 & 1 & & & & \\
\hline Cerebellum ${ }^{c}$ & 1/n.a./0 & $1 / 1 / 1$ & & & & \\
\hline \multicolumn{7}{|l|}{ Spinal cord } \\
\hline Anterior horn & 2 & 1 & & & & \\
\hline IML & 2 & 0 & & & & \\
\hline Clarke's column & 0 & 0 & & & & \\
\hline Posterior horn & 2 & 1 & & & & \\
\hline Wm & 0 & 1 & & & & \\
\hline \multirow[t]{2}{*}{ Others } & Ependymal cell & Choroid plexus & & & & \\
\hline & 0 & 2 & & & & \\
\hline
\end{tabular}

The density of inclusions was graded according to the percentage of inclusion-bearing cells: 0 , none; $1,0-10 \% ; 2,10-40 \% ; 3,>40 \%$

${ }^{\mathrm{a}}$ cortex/white matter; ${ }^{\mathrm{b}} \mathrm{CA} 1 / \mathrm{CA} 4 /$ dentate gyrus; ${ }^{\mathrm{C}}$ Cerebellar cortex/white matter/dentate nucleus; wm, white matter; n.a., not available; NBM, nucleus basalis of Meynert; i/e, internal segment/external segment; nuc, nucleus; IO, inferior olive; IML, intermediolateral cell column; ggl, ganglion; EC, endothelial cell; SMC, smooth muscle cell

Table 2 Intranuclear inclusions in diseases associated with noncoding CGG repeat expansions

$\begin{array}{lll}\begin{array}{l}\text { NIID1 [4, 5] } \\ \text { NBPF19 }\end{array} & \begin{array}{l}\text { OPML1 [2] } \\ \text { LOC642361/ } \\ \text { NUTM2B-AS1 }\end{array} & \begin{array}{l}\text { OPDM1 } \\ \text { Present study } \\ \text { LRP12 }\end{array} \\ \begin{array}{lll}\text { Undetectable } & \text { n.a. } & \text { Undetectable } \\ \text { n.a. } & \text { n.a. } & \text { Tubulofilamentous } \\ \text { Widespread } & \text { n.a. } & \text { Widespread } \\ \text { Fine filamentous } & \text { n.a. } & \text { Fine filamentous }\end{array}\end{array}$

OPDM oculopharyngodistal myopathy, OPML oculopharyngeal myopathy with leukoencephalopathy, NIID neuronal intranuclear inclusion disease, n.a. not available 
genetically confirmed autopsy case showing histological evidence of systemic NIID-1 like lesions. Further clinicopathological and molecular studies will help to clarify how noncoding CGG expansions in different genes form the NIID-OPDM spectrum, and the pathomechanism underlying the similarities and differences among these disorders.

\section{Supplementary information}

Supplementary information accompanies this paper at https://doi.org/10 1186/s40478-020-00945-2.

\section{Additional file 1}

\section{Abbreviations}

OPDM: Oculopharyngodistal myopathy; NIID: Neuronal intranuclear inclusion disease; OPML: Oculopharyngeal myopathy with leukoencephalopathy; RV: Rimmed vacuole

\section{Authors' contributions}

RS, HS and AK designed research project, and performed pathological analysis and drafted the manuscript for intellectual content. TM, NM, KS, and $\mathrm{YH}$ collected clinical data. $\mathrm{NH}, \mathrm{YH}, \mathrm{AM}$ and $\mathrm{TI}$ designed the molecular experiments and performed those. IK and $\mathrm{OO}$ discussed the results and commented on the manuscript text. The authors read and approved the final manuscript.

\section{Funding}

This work was supported in part by JSPS grants-in aid for Scientific Research to RS (19 K21314), HS (19 K07841), IK (17 K09776), OO (JP26117006), and AK (19H01061 and 19H05559), and AMED to TI (JP20dk0207045).

\section{Availability of data and materials}

The datasets used and analysed during the current study available from the corresponding author on reasonable request.

\section{Ethics approval and consent to participate}

The present study was approved by the Ethics Committee of Niigata University (G2019-0020). Written informed consent for autopsy including the use of tissues for research purposes was obtained from the patients' family.

\section{Consent for publication}

Family members have consented to publication.

\section{Competing interests}

The authors declare that they have no competing interests.

\section{Author details}

${ }^{1}$ Department of Pathology, Brain Research Institute, Niigata University, 1-757 Asahimachi, Chuo-ku, Niigata 951-8585, Japan. ²Department of Neurology, Sado General Hospital, Niigata, Japan. ${ }^{3}$ Department of Neurology, Brain Research Institute, Niigata University, Niigata, Japan. ${ }^{4}$ Department of Molecular Genetics, Brain Research Institute, Niigata University, Niigata, Japan. ${ }^{5}$ Comprehensive Medical Education Center, Niigata University School of Medicine, Niigata, Japan

Received: 30 March 2020 Accepted: 7 May 2020

Published online: 03 June 2020

\section{References}

1. Durmus H, Laval SH, Deymeer F, Parman Y, Kiyan E, Gokyigiti M et al (2011) Oculopharyngodistal myopathy is a distinct entity: clinical and genetic features of 47 patients. Neurology 76:227-235

2. Ishiura H, Shibata S, Yoshimura J, Suzuki Y, Qu W, Doi K et al (2019) Noncoding CGG repeat expansions in neuronal intranuclear inclusion disease, oculopharyngodistal myopathy and an overlapping disease. Nat Genet 51:1222-1232
3. Lu H, Luan X, Yuan Y, Dong M, Sun W, Yan C (2008) The clinical and myopathological features of oculopharyngodistal myopathy in a Chinese family. Neuropathology 28:599-603

4. Sone J, Mitsuhashi S, Fujita A, Mizuguchi T, Hamanaka K, Mori K et al (2019) Long-read sequencing identifies GGC repeat expansions in NOTCH2NLC associated with neuronal intranuclear inclusion disease. Nat Genet 51:12151221

5. Sone J, Mori K, Inagaki T, Katsumata R, Takagi S, Yokoi S et al (2016) Clinicopathological features of adult-onset neuronal intranuclear inclusion disease. Brain 139:3170-3186

6. Sun QY, Xu Q, Tian Y, Hu ZM, Qin LX, Yang JX et al (2020) Expansion of GGC repeat in the human-specific NOTCH2NLC gene is associated with essential tremor. Brain 143:222-233

7. Thevathasan W, Squier W, Maclver DH, Hilton DA, Fathers E, Hilton-Jones D (2011) Oculopharyngodistal myopathy - a possible association with cardiomyopathy. Neuromuscul Disord 21:121-125

\section{Publisher's Note}

Springer Nature remains neutral with regard to jurisdictional claims in published maps and institutional affiliations.
Ready to submit your research? Choose BMC and benefit from:

- fast, convenient online submission

- thorough peer review by experienced researchers in your field

- rapid publication on acceptance

- support for research data, including large and complex data types

- gold Open Access which fosters wider collaboration and increased citations

- maximum visibility for your research: over $100 \mathrm{M}$ website views per year

At BMC, research is always in progress.

Learn more biomedcentral.com/submissions 